\title{
A Mobile Acquisition System and a Method for Hips Sway Fluency Assessment
}

\author{
Andrea Civita ${ }^{1,+}{ }^{,}$Simone Fiori ${ }^{2, *,+}+\mathbb{C}$ and Giuseppe Romani ${ }^{1,+}$ \\ 1 School of Information and Automation Engineering, Università Politecnica delle Marche (uPM), \\ 60131 Ancona, Italy; andreacivita994@gmail.com (A.C.); gromani14@gmail.com (G.R.) \\ 2 Dipartimento di Ingegneria dell'Informazione, Università Politecnica delle Marche (uPM), 60131 Ancona, Italy \\ * Correspondence: s.fiori@univpm.it \\ + These authors contributed equally to this work.
}

Received: 31 October 2018; Accepted: 10 December 2018; Published: 12 December 2018

\begin{abstract}
The present contribution focuses on the estimation of the Cartesian kinematic jerk of the hips' orientation during a full three-dimensional movement in the context of enabling eHealth applications of advanced mathematical signal analysis. The kinematic jerk index is estimated on the basis of gyroscopic signals acquired offline through a smartphone. A specific free mobile application is used to acquire the gyroscopic signals and to transmit them to a personal computer through a wireless network. The personal computer elaborates the acquired data and returns the kinematic jerk index associated with a motor task. A comparison of the kinematic jerk index value on a number of data sets confirms that such index can be used to evaluate the fluency of hips orientation during motion. The present research confirms that the proposed gyroscopic data acquisition/processing setup constitutes an inexpensive and portable solution to motion fluency analysis. The proposed data-acquisition and data-processing setup may serve as a supporting eHealth technology in clinical bio-mechanics as well as in sports science.
\end{abstract}

Keywords: complex body movement; hips sway; fluency assessment; gyroscopic sensors; smartphone

\section{Introduction}

Complex body tasks, such as rock climbing, involve the alternation of periods dedicated to postural regulation and of quadruped displacement. Figure 1 illustrates a typical hips sway during climbing. In neurosciences [1] and in sports science [2], the assessment of body fluency provides valuable information on the patient's musculoskeletal system as well as of the mental state of an athlete (for example, rigid posture and erratic movements during a climbing performance may indicate a state of anxiety [3]); likewise, since restoring movement fluency is a key focus in physical rehabilitation, objective measurement of fluency is of prime importance [4]. In these kinds of experiments, it is important to investigate how the affordances of an indoor climbing wall changes for intermediate climbers following a period of practice [5].

Hips displacement is one of the indicators of osteoarthritis in athletes [6]. While previous studies on the fluency of body movements from temporal and spatial measurement analyses took into account only the displacement of the hips on a two-dimensional projective plane [7], recent studies have highlighted how the presence of anteroposterior and lateral sway would rather imply the need of combined temporal and full three-dimensional movement analysis. Such three-dimensional movement analysis should take into account both hip translation and rotation. The recent study [3], in particular, focuses on the assessment of the kinematic jerk of the hips rotation during a complex body task. In kinematics, the jerk of a positional 
variable is defined as its third time-derivative, namely, as the derivative of the acceleration. Other kinematic indicators, such as mechanical power, were made use of in order to establish the level of expertise of a climber [8].

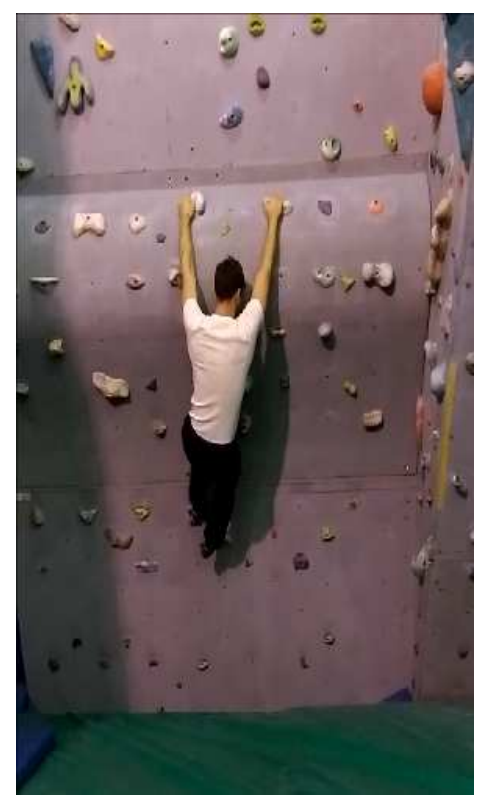

Figure 1. Illustration of an indoor wall-climbing setup used to evaluate the motion fluency of an athlete during a complex body task. The picture illustrates, in particular, the hip sway during climbing as an instance of postural regulation. This picture represents a single frame from a series of three longer video clips acquired during this research in a gymnasium equipped with indoor wall-climbing facilities and by the help of three professional male athletes, whose performances will be evaluated in Section 4.2.

The hips orientation is described by a $3 \times 3$ rotation matrix, which is a function of the time. Therefore, the orientation jerk is defined as the third-order time-derivative of a curve on the group of special orthogonal matrices. Such group, denoted as $\mathrm{SO}(3)$, is a curved manifold, hence, in such space, the assessment of kinematic variables such as acceleration and jerk is not straightforward as it is for curves in the ordinary space $\mathbb{R}^{3}$.

Although a complete analysis of body movement fluency would imply a statistical characterization of both hips' rotation jerk and translation jerk, the study [3] showed that the (normalized) kinematic jerk of hip orientation and the (normalized) kinematic jerk of hips translation exhibit a significant positive correlation, signifying that both measures provide a similar measure of fluency. In addition, several clinical studies focus on hip rotation only, such as, for example, the research [9] that focuses on the analysis of reduced range of motion of the hips, which is an established clinical indicator of osteoarthritis.

A number of related studies made use of the kinematic jerk of positional data to investigate bio-mechanical as well as industrial mechanics phenomena. In the studies [1,10], concerning human three-dimensional finger-pointing movements, it is assumed that the speed profile along the hand path is determined by minimization of the squared kinematic jerk. Likewise, in the paper [11], the impact of a kinematic jerk index is analyzed in the context of the generation of minimum time collision-free trajectories for industrial robots in a complex environment. In the research paper [12], it was hypothesized that there exists a functional relationship between kinematic jerk cost and energy consumption during walking. Energy consumption during walking is closely related to the gait speed. Minimum kinematic jerk theory states that a body movement is carried out by minimizing the kinematic jerk of the motion trajectory. 
The study [13] in earthquake science showed that kinematic jerk and its response spectrum can enhance the recognition of a non-stationary ground motion. In fact, kinematic jerk represents the non-stationary component in the high frequency band of an earthquake wave.

Recently, the scientific research conducted in the area of eHealth has benefited from advanced mathematical methods in signal processing and data analysis as well as from new wearable, inexpensive sensory devices. Nowadays, smartphones come with a large set of embedded sensors, including gyroscopes. Ref. [14] has made evident how new accessible methods delivered through mobile mixed reality are facilitating human learning via interactive mobile environments. The article [15] proposes a handover algorithm that guarantees seamless connectivity in wireless body area networks (WBANs), which have become an emerging technology in eHealth-care, where patients are equipped with wearable body sensor nodes to gather sensory information for remote monitoring. The previous contribution [16] investigated instrumented 'Timed Up and Go' based on a specialized measurement system, namely, the accelerometer of a smartphone, to identify and evaluate specific mobility skills. The contribution [17] evaluated the reliability of a smartphone accelerometer in gait analysis. Likewise, the project SmartGait ${ }^{\mathrm{TM}}$ [18] makes use of a smartphone to acquire a number of postural indicators from a moving body with the aim to develop an inexpensive and accurate gait tracking tool that can be easily implemented by researchers and clinicians. In the paper [19], it is observed that the storing position of a smartphone impacts the performance of the notification to a user and the measurement of embedded sensors, which plays an important role in a device's functionality control; in this article, different storing positions are recognized using the accelerometer of the smartphone.

It is also interesting to mention how the assessment of complex body parts movement has recently found plenty of worthy applications. Ref. [20] deals with applications in modeling expressive gestures in multimodal interaction and in the development of multimodal interactive systems by explicitly taking into account the role of non-verbal expressive gesture in the communication process. Ref. [21] suggests a way to assess the expertise level of a surgeon by evaluating the hand motions, based on the assumption that hand motions made by a surgeon may reflect his skills. Ref. [22] presents a mobile application that provides activity data from smartphone sensors and applies experience based sampling to collect the patients' subjective perceptions, in the context of remote monitoring of cancer-related fatigue.

The aim of the present contribution is to illustrate the details of smartphone-based acquisition systems to evaluate hip rotation fluency by estimating the kinematic jerk of the acquired signals. In particular, the present paper proposes:

- a simple, inexpensive measurement setting based on a smartphone equipped with a free software application that reads the outcomes of the sensors embedded in the smartphone and transmits the readings to a personal computer via a wireless network. The necessary hardware is commonly available in every environment, including private houses, gymnasiums, clinics and public spaces.

- $\quad$ a specific MATLAB ${ }^{\circledR}$ software (made by the authors) that is able to read the data stream from a wireless network and to compute the kinematic jerk index associated with a body movement session, with a minimal amount of user setting effort. The developed MATLAB ${ }^{\circledR}$ software is made available within the paper for easy replicability.

The obtained experimental results, conducted on real-world data sets, confirm that the fluency evaluation procedure is reliable and the numerical results of kinematic jerk index estimation confirm the self-evaluation of the athletes' skills.

The present paper is organized as follows. Section 2 explains relevant details about the acquisition of a gyroscopic data-stream by a smartphone and a personal computer, the actual setting that was used in the present research to acquire the orientation data during wall climbing. Section 3 treats in detail the computational aspects concerning the estimation of a kinematic jerk index associated with a gyroscopic 
data stream. Section 4 illustrates features of the proposed method on a number of real-world gyroscopic datasets. Section 5 concludes the paper.

\section{Acquisition of a Gyroscopic Data-Stream by a Smartphone and a Personal Computer}

Physical attitude of an athlete during a performance may be measured through a variety of sensors, including inertial measurement units as well as eye tracking systems [23]. The present section explains how to connect wirelessly an ANDROID ${ }^{\text {TM}}$-based smartphone with a personal computer equipped with a MATLAB ${ }^{\circledR}$ development environment to process the acquired data.

The smartphone runs under an ANDROID ${ }^{\mathrm{TM}}$ mobile operating system and is worn on the waist of an athlete or a patient. An installed mobile application, namely the Hyper IMU mobile application (available for ANDROID ${ }^{\mathrm{TM}}$ [24]), version 1.3, acquires the orientation of the smartphone expressed by three angles, namely yaw, ranging in $[0,360)$, pitch, ranging in $[-180,180)$ and roll, ranging in $[-90,90]$. An illustration of the three angles is displayed in Figure 2.

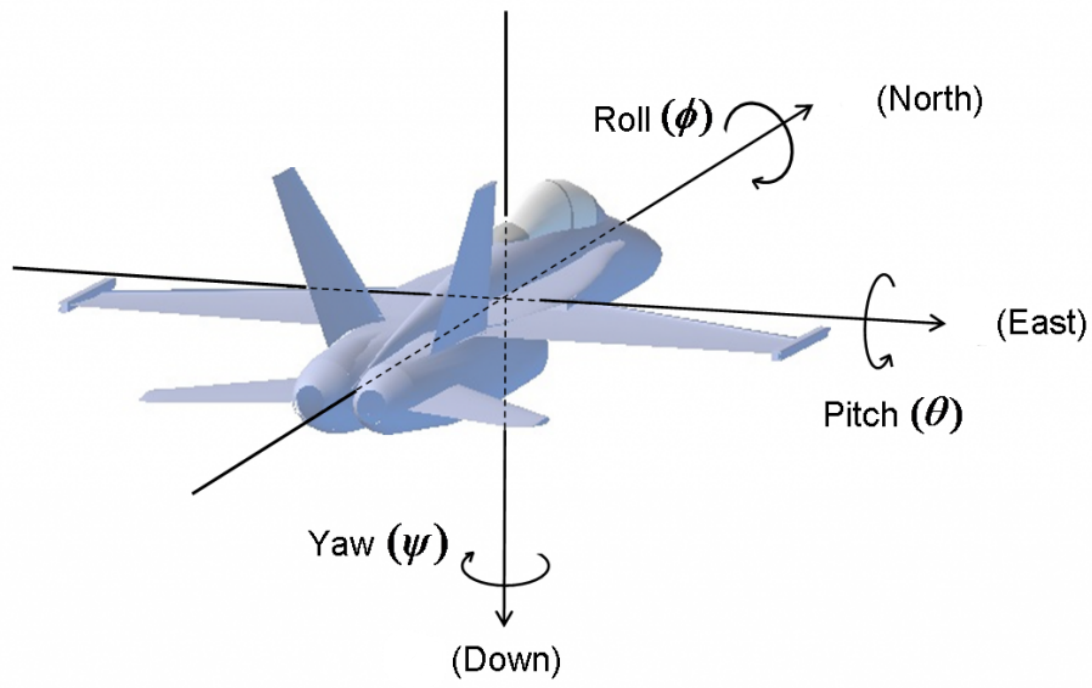

Figure 2. An illustration of the three angles yaw, pitch and roll returned by the 'Hyper IMU' mobile application. This set of three angles, common in aeronautics, is widely adopted in mobile applications to describe the orientation of a smartphone.

The application allows the user to set the sampling period from a minimum value of $\Delta T=20 \mathrm{~ms}$. A screenshot of the Hyper IMU mobile application is displayed in Figure 3.

The transmission of the data-stream from a smartphone to a MATLAB ${ }^{\circledR}$-equipped personal computer takes place through a User Datagram Protocol (UDP, RFC 768) [25]. The UDP was defined to afford a simple packet-switched communication mode in networks of interconnected devices. The UDP assumes that the Internet Protocol (IP) is used as underlying protocol and provides a means for mobile applications and computer programs to exchange messages with each other with a minimum of protocol machinery (and is therefore suitable for communication networks where security and data protection is not an issue). 


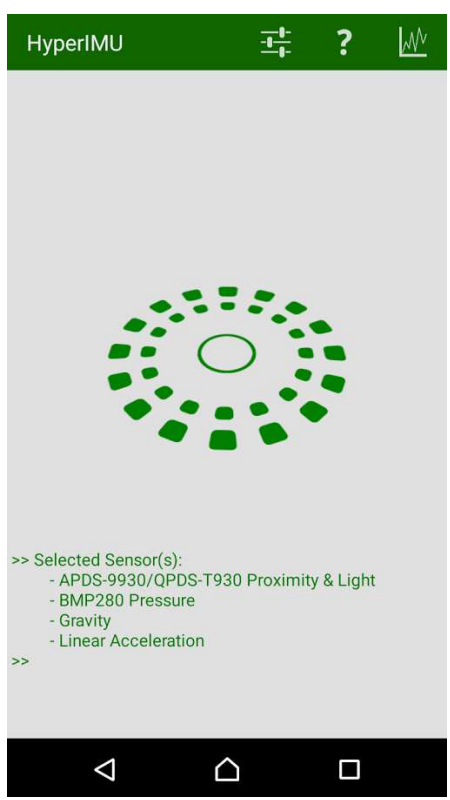

Figure 3. A screenshot of the freeware "Hyper IMU" mobile application for the ANDROID ${ }^{\mathrm{TM}}$ mobile operating system [24].

If the environment where the measurements take place is served by a wireless router, the smartphone and the personal computer may be connected to the wireless network and exchange data. Otherwise, as, for example, in an outdoor experiment, it is possible to make a personal computer behave as a wireless-network access point that the smartphone may bind to. Under a MICROSOFT ${ }^{\circledR}$ WINDOWS ${ }^{\circledR}$ operating system (Redmond, WA, USA), the access point may be created upon the execution of the command

netsh wlan set hostednewtork mode allow

ssid=... key=... keyUsage=persistent,

where the parameter ssid denotes the name assigned to the access point and the parameter key denotes the access password. The wireless network starts operating upon the execution of the command

netsh wlan start hostednetwork,

while, upon the execution of the command

netsh wlan stop hostednetwork,

the access point stops operating.

The MATLAB ${ }^{\circledR}$ platform was used to acquire gyroscopic signals from a smartphone and to compute its kinematic jerk index. In the MATLAB ${ }^{\circledR}$ language, a port is managed like a data-file; therefore, reading out signals from a smartphone via a wireless network is equivalent to reading the records of a data-file, as shown in the code excerpt Listing 1. 
Listing 1. MATLAB ${ }^{\circledR}$ script to acquire the gyroscopic signals from a smartphone via a wireless network.

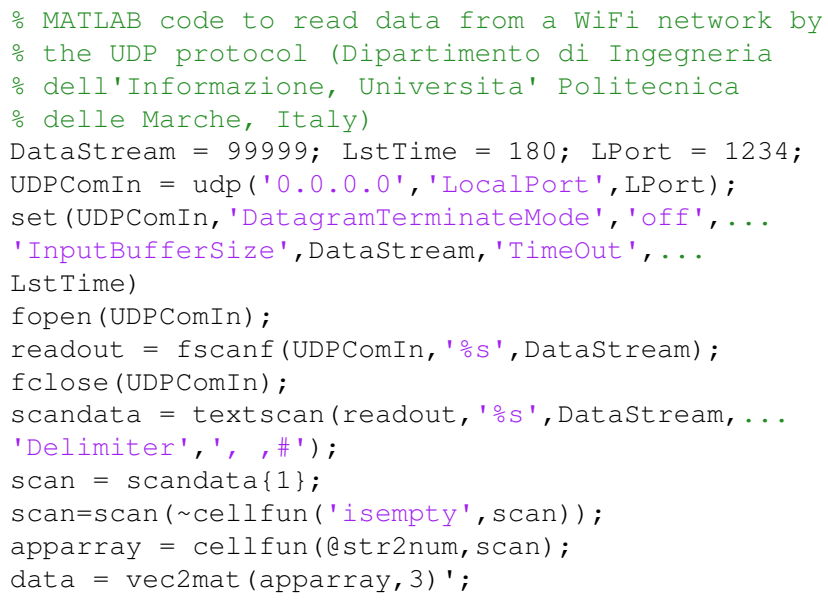

Such script shows the minimal instructions set necessary to acquire the data correctly. It might need additional instructions to reset the MATLAB ${ }^{\circledR}$ settings (such as the instrreset command) and to display and save the acquired data.

The Line 6 of the MATLAB ${ }^{\circledR}$ script creates the UDP object UDPComIn and specifies the IP address and the port number LPort of the transmitter. Setting the IP address to the conventional value 0.0.0.0 indicates that the actual IP address of the transmitting device is not specified and that the computer will listen to any data stream sent through the port specified at Line 5 (which needs, therefore, to be uniquely dedicated to the communication between the smartphone and the personal computer). Using the conventional IP address 0.0.0.0 allows the acquisition script in Listing 1 to work within a dynamic host configuration protocol (DHCP) network.

Line 7 of the script sets relevant attributes of the object UDPComIn, namely:

- the attribute UDPComIn.DatagramTerminateMode to the value off, in order to make the personal computer keep acquiring packets as they become available from the smartphone;

- the attribute UDPComIn. InputBufferSize to the value DataStream, whose value is set in Line 5, so that the personal computer will stop listening after a sufficiently large number of acquired bytes;

- the attribute UDPComIn. TimeOut to the value LstTime, expressed in seconds and set in Line 5, so that the personal computer will stop listening after a sufficient time-span to allow acquiring the gyroscopic signals pertaining to a sufficiently long experiment.

It should be noted that the conditions set on the input buffer size and on the timeout cause the listening process to stop, whichever is verified first.

Line 11 of the MATLAB ${ }^{\circledR}$ script Listing 1 reads the character-string output from the smartphone into the variable readout. The variable readout appears as a string of the format

Yaw $_{1}$, Pitch $_{1}$, Roll $_{1} \# Y_{2}$, Pitch $_{2}$, Roll $_{2} \# Y_{3}$, Pitch $_{3}$, Roll $_{3} \ldots$

Namely, every record is formed by three angles (expressed in degrees and represented as floats) separated by two commas and each record ends with a hash character.

The commands in Lines 13-15 separate each record and construct a cell object, named scan, that contains the list of acquired yaw, pitch and roll angles. Line 16 expunges empty records. 
Line 17 converts the string-type cell elements into numbers and Line 18 constructs a $3 \times N$ array data whose columns contain the acquired angles.

A further auxiliary MATLAB ${ }^{\circledR}$ script converts the yaw, pitch and roll angles triples to a gyroscopic signal $R(t)$, whose elements are $3 \times 3$ rotation matrices, that represents the instantaneous orientation of the smartphone with respect to a fixed reference frame (typically defined on the basis of a magnetoscopic sensor). The obtained gyroscopic signal will be converted back to angular speeds as explained in the next section. The intermediate rotation data are introduced in order to make the procedure independent of the type of acquired data, since different gyroscopic mobile applications might acquire different kinds of angles. (The proper Euler angles triples and the Tait-Bryan angles triples amount to twelve possible combinations [26].)

\section{Computational Estimation of the Kinematic Jerk Index Associated with a Gyroscopic Data Stream}

Given a hip rotation trajectory $R(t)$, we define the "angular velocity" matrix field as

$$
\Omega(t)=R^{T}(t) \frac{d R(t)}{d t} .
$$

The matrix $\Omega$ is $3 \times 3$ and skew-symmetric, namely, its diagonal elements are zero and only its three strictly-upper triangular entries are independent of each other, namely

$$
\Omega(t)=\left[\begin{array}{ccc}
0 & \omega_{a}(t) & \omega_{b}(t) \\
-\omega_{a}(t) & 0 & \omega_{c}(t) \\
-\omega_{b}(t) & -\omega_{c}(t) & 0
\end{array}\right] .
$$

The "vee" operator ${ }^{\vee}$ [27] returns, in a vector, such independent entries. One may, thus, associate with a trajectory $R(t)$ a vectorized angular velocity

$$
\omega(t) \stackrel{\text { def }}{=} \Omega^{\vee}(t)=\left[R^{T}(t) \dot{R}(t)\right]^{\vee},
$$

whose entries are $\omega(t)=\left[\omega_{a}(t) \omega_{b}(t) \omega_{c}(t)\right]^{T}$.

The contribution [3] takes, as hip rotation jerk, the second-order time-derivative of the $3 \times 1$ vector $\omega$, namely $\ddot{\omega}(t)$. The advantage of such definition of kinematic jerk is that it appears as a vector in $\mathbb{R}^{3}$ and the scalar kinematic jerk may be simply computed through the L2-norm $\|\ddot{\omega}\|$. By using the above parameterization for the angular velocity matrix, the scalar kinematic jerk taken in [3] is computed as

$$
\|\ddot{\omega}\|=\sqrt{\ddot{\omega}_{a}^{2}+\ddot{\omega}_{b}^{2}+\ddot{\omega}_{c}^{2}}
$$

The integrated kinematic jerk index associated with a trajectory $R(t), t \in[0 T]$, is defined as

$$
J \stackrel{\text { def }}{=} C \int_{0}^{T}\|\ddot{\omega}\| d t
$$

where the constant $C$ is chosen in a way that makes the kinematic jerk index $J$ dimensionless. The index $J$ defined in Label (5) is named Cartesian kinematic jerk to distinguish it from other possible definitions (see, for example, [28]). Furthermore, the constant $C$ is chosen in a way that makes the kinematic jerk index independent from the total length of a path and from the total observation time $T$.

In order to estimate numerically the derivative $\dot{R}(t)$, Ref. [3] makes implicit use of the following idea. Let $R_{k}$ and $R_{k+1}$ denote two subsequent samples of an acquired, discrete-time gyroscopic data-stream 
counting $N$ observations spaced apart in a time of $\Delta T$ seconds. The relationship between the number of acquired samples, the sampling interval and the total observation time is

$$
T=(N-1) \Delta T
$$

Consider such samples as joined by a geodesic arc of the curved space $\mathrm{SO}$ (3) (for a review of the geometry of the special orthogonal group, readers might consult, e.g., [29,30]), namely

$$
R_{k+1}=\exp _{R_{k}}\left(\Delta T \cdot R_{k} \cdot \Omega_{k}\right)=R_{k} \operatorname{Exp}\left(\Delta T \cdot \Omega_{k}\right),
$$

where $\exp _{R}(\cdot)$ denotes a group exponential map at $R \in \mathrm{SO}(3)$ and Exp denotes the matrix exponential. Reversing such formula by means of the logarithmic map, one gets the estimate

$$
\Omega_{k}=\frac{1}{\Delta T} \log \left(R_{k}^{T} R_{k+1}\right), k=0,1, \ldots, N-1,
$$

where Log denotes the (principal) matrix logarithm (that can be calculated, by the MATLAB ${ }^{\circledR}$ language, by the function logm as long as its argument is sufficiently close to the identity, namely, as long as two subsequent samples $R_{k}$ and $R_{k+1}$ are sufficiently close to one another). Let us define $\omega_{k} \stackrel{\text { def }}{=} \Omega_{k}^{\vee}$. Now, the kinematic jerk index may be estimated as the sum

$$
\tilde{J} \stackrel{\text { def }}{=} C \Delta T \sum_{k=2}^{N-1}\left\|\ddot{\omega}_{k}\right\|,
$$

where

$$
\ddot{\omega}_{k} \stackrel{\text { def }}{=} \frac{\omega_{k}-2 \omega_{k-1}+\omega_{k-2}}{(\Delta T)^{2}} .
$$

The total length of a measured data-stream may be defined as

$$
d \stackrel{\text { def }}{=} \int_{0}^{T}\|\omega\| d t
$$

which can be estimated numerically as

$$
\tilde{d} \stackrel{\text { def }}{=} \Delta T \sum_{k=0}^{N-1}\left\|\omega_{k}\right\|
$$

In the spirit of the contributions $[3,28]$, the normalization constant $C$ may be defined as

$$
C \stackrel{\text { def }}{=} \frac{(N-2)^{2} \Delta T^{2}}{\tilde{d}} .
$$

The MATLAB ${ }^{\circledR}$ function that implements the above calculations is displayed in Listing 2. 
Listing 2. MATLAB ${ }^{\circledR}$ function to calculate the Cartesian kinematic jerk.

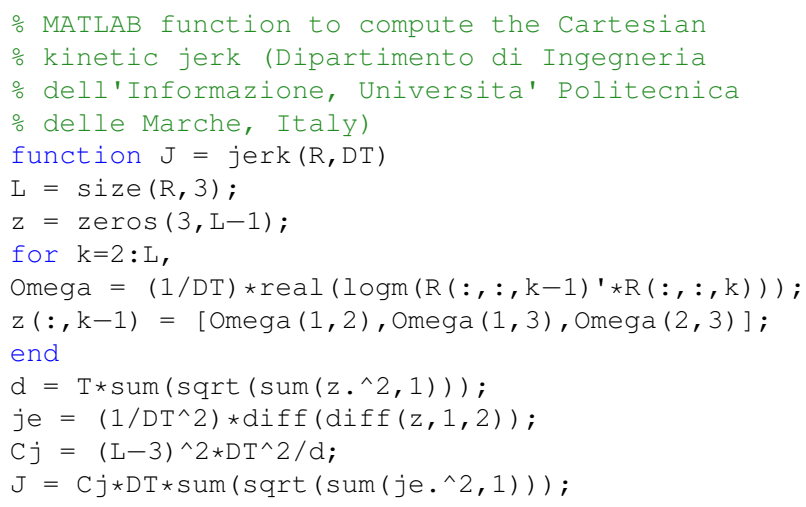

The MATLAB ${ }^{\circledR}$ function displayed in Listing 2 inputs an array $R$ of size $3 \times 3 \times N$ and a sampling period DT and returns the numerical estimate of the Cartesian kinematic jerk index (9). Note that at Line 9 the operator real was introduced to get rid of the imaginary part of the matrix logarithm, that, in principle, should be equal to zero, while, in practice, might slightly differ from zero because of finite-precision machine calculations.

\section{Experimental Results}

The current section presents results of experiments on the acquisition of hip rotation data and on the computational assessment of the fluency of a body movement during complex motor tasks. The experiments were performed on acquired data measured in different conditions.

\subsection{Acquisition and Evaluation of Test-Type Complex-Body-Movement Signals}

As a preliminary test of the described acquisition procedure and of the kinematic jerk-estimation scripts, three different kinds of gyroscopic signals were acquired during three complex body tasks: walking, running and jumping. Everyday phisical activities such as sitting on chair/floor and brisk/slow walking are the subject of human activity recognition as a part of ongoing reearch endeavors in medical and surveillance applications [31]. The "walking" and "running" body tasks were carried out over a straight path 10 meters long, while the "jumping" body task was performed on site. The running performance is known to be an indicator of knee osteoarthritis [32]. During the acquisition of the gyroscopic signals, the smartphone was fastened to the subject's belt through an elastic band.

A number $N=450$ of samples were acquired for each task. The data stream acquired during walking is displayed in Figure 4, the data stream acquired during running is displayed in Figure 5 and the data stream acquired during jumping is displayed in Figure 6. 

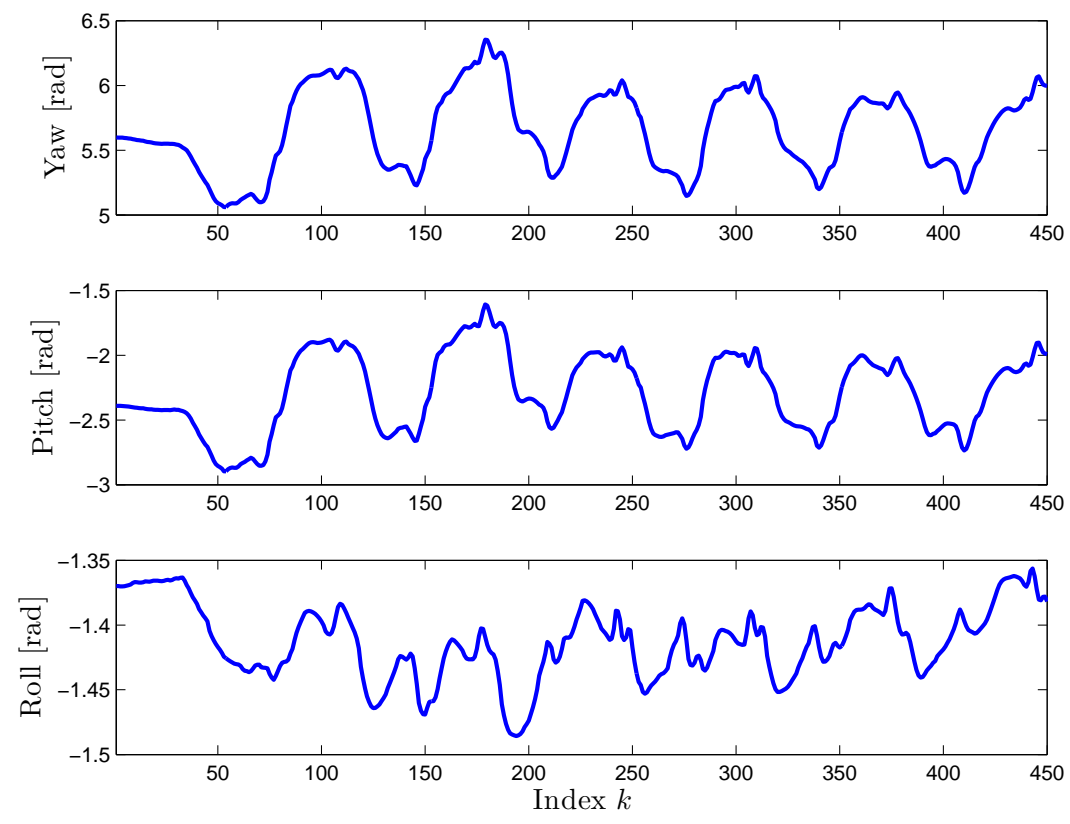

Figure 4. Graphical representation of the Yaw, Pitch and Roll angles acquired during walking (unwrapped, radians).
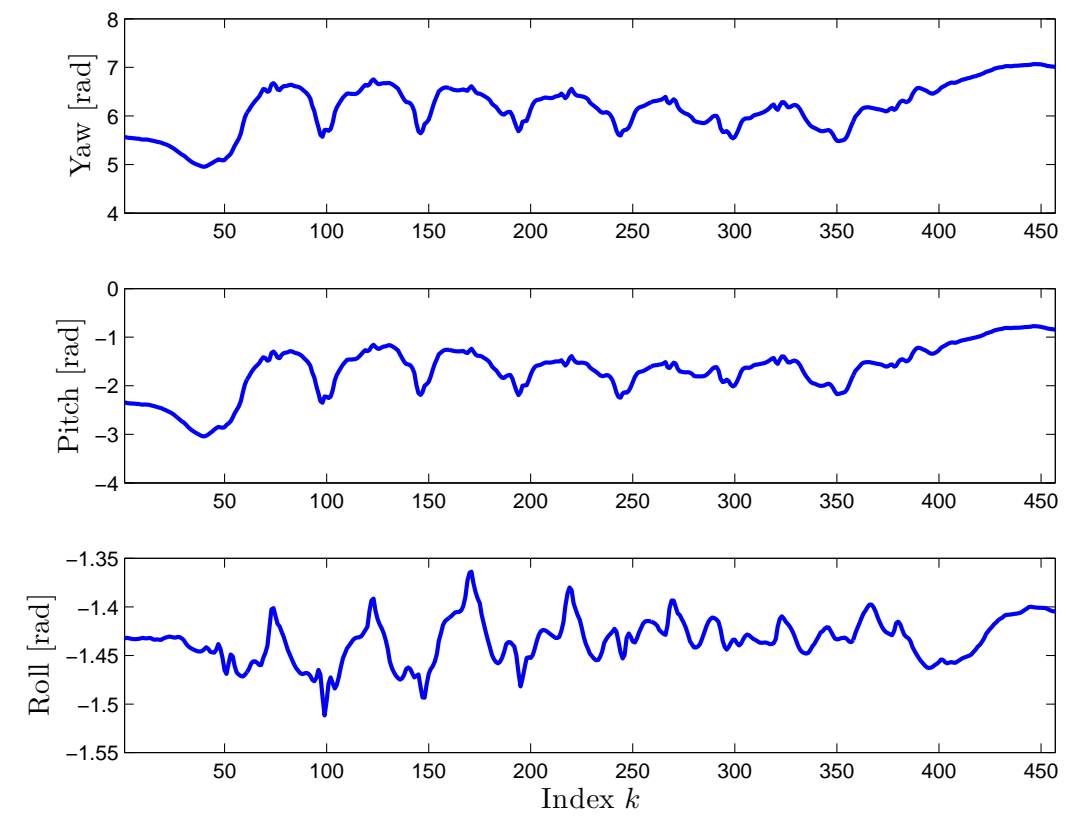

Figure 5. Graphical representation of the Yaw, Pitch and Roll angles acquired during running (unwrapped, radians). 

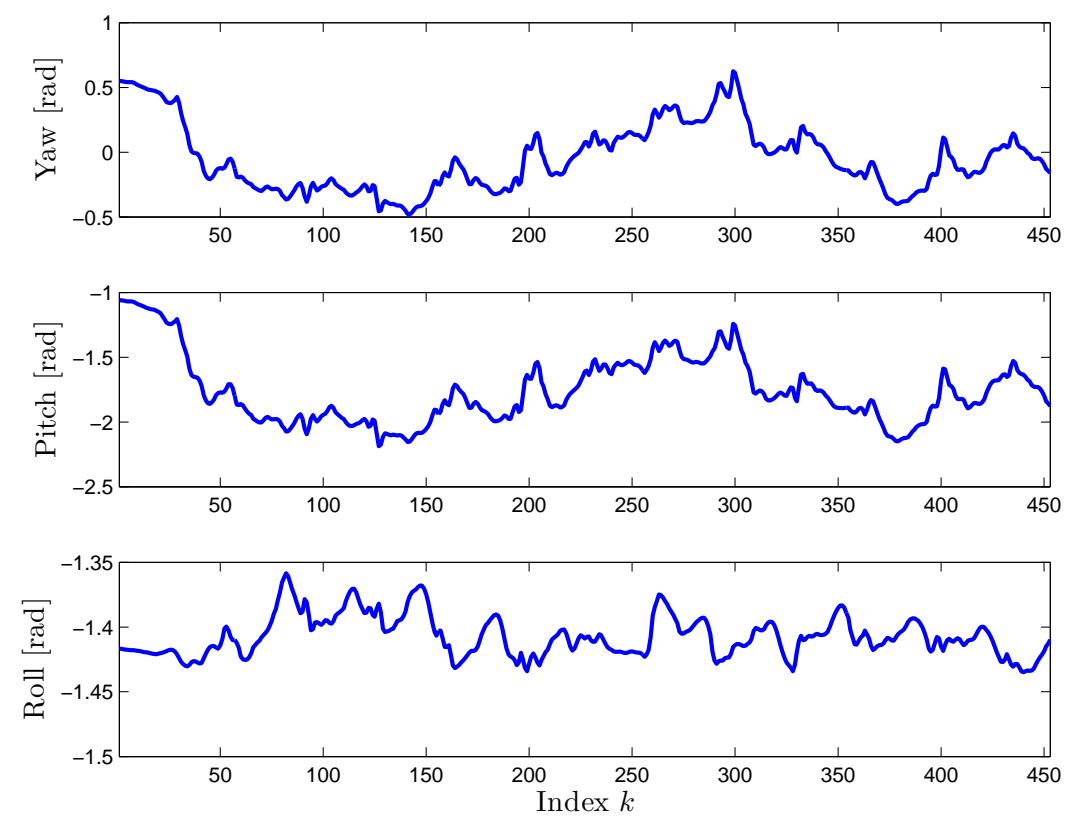

Figure 6. Graphical representation of the Yaw, Pitch and Roll angles acquired during jumping (unwrapped, radians).

The results of the computation of the Cartesian kinematic jerk $\tilde{J}$ according to Formula (9) are displayed in Table 1. As it is readily appreciated, the Cartesian kinematic jerk grows in value as the analyzed motion becomes increasingly erratic.

Table 1. Cartesian kinematic jerk calculated on acquired gyroscopic signals of the type "Walk", "Run" and "Jump".

\begin{tabular}{cc}
\hline & Cartesian Kinematic Jerk \\
\hline Walking & 104,517 \\
Running & 158,509 \\
Jumping & 206,153 \\
\hline
\end{tabular}

\subsection{Acquisition and Evaluation of Signals during Indoor Wall Climbing Sessions}

The gyroscopic signals pertaining to the present experiment were acquired in a gymnasium equipped with a professional wall-climbing facility and the signals were acquired by the help of three male athletes each on a different training and skill level.

In particular, an Expert, an Intermediate-level and an Inexpert climbers completed two different climbing tasks, hereafter termed Task $\alpha$ and Task $\beta$, corresponding to two different kinds of walls. A frame of a camera-recording taken during a climbing session is displayed in Figure 1. The experience level of the athletes as well as the difficulty level of a climbing task were evaluated by a professional trainer.

Before performing each task, the athletes planned a sequence of hand-grasps, in order to make the execution of the tasks as uniform as possible, while the foot standing strategy was left to each athlete's discretion. Nevertheless, due to the different level of experience of the athletes, the elapsed time took by each athlete to complete the same task was different, resulting in a different data-stream length for each of the six resulting sessions (one session per athlete, per task).

As an example of the acquired signals over Task $\beta$, the acquired gyroscopic signals, under the form of Yaw, Pitch and Roll pertaining to the Inexpert athlete are displayed in Figure 7, the acquired 
signals pertaining to the Intermediate-level athlete are displayed in Figure 8, while the gyroscopic signals pertaining to the Expert athlete are displayed in Figure 9.
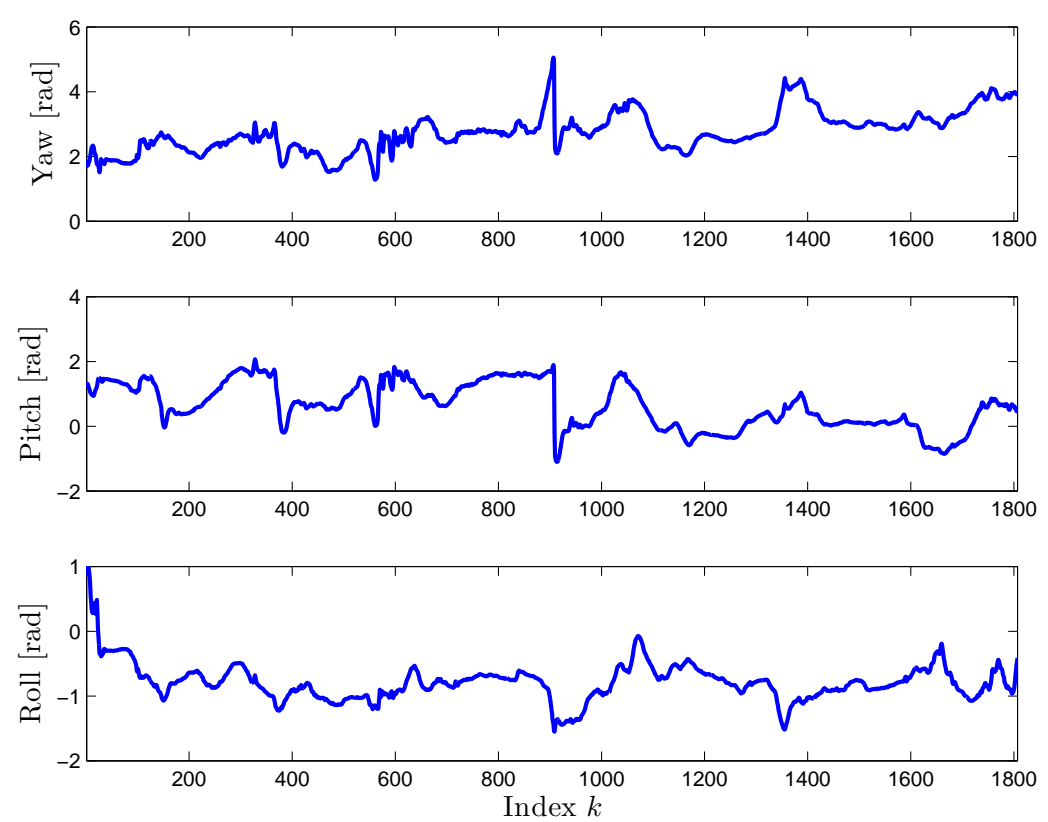

Figure 7. Gyroscopic signals acquired in a gym equipped with a professional wall-climbing facility: inexpert athlete on Task $\beta$ (unwrapped, radians).
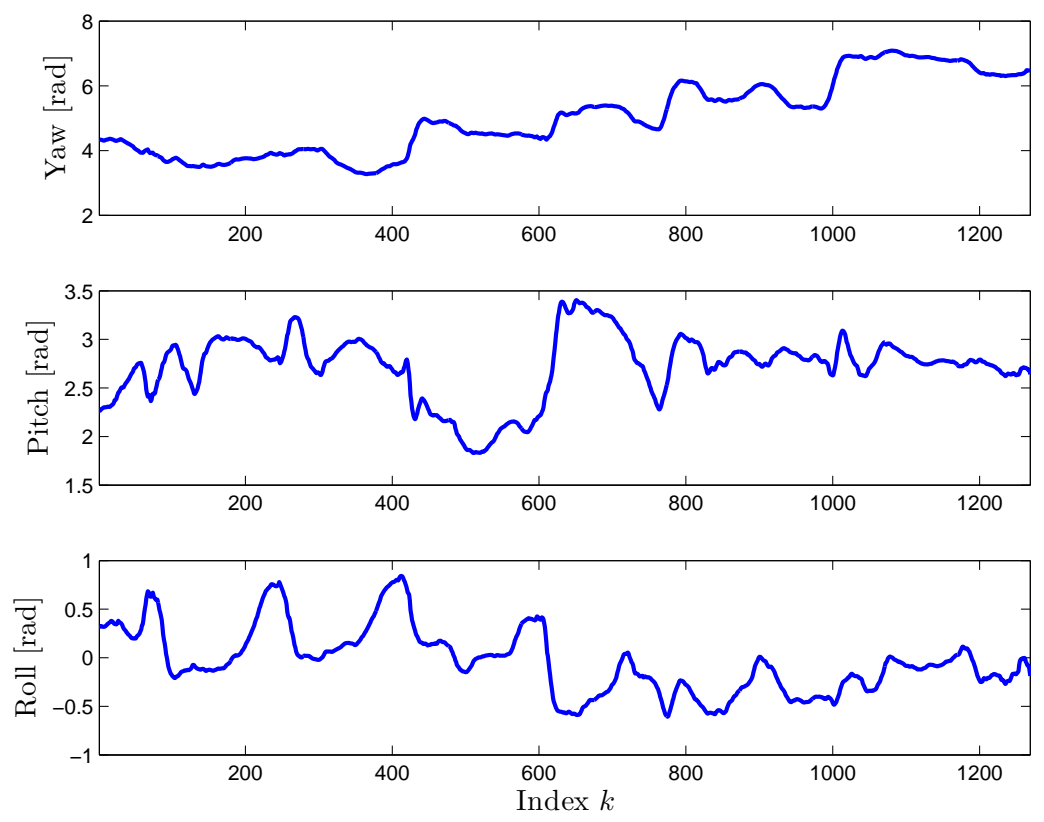

Figure 8. Gyroscopic signals acquired in a gym equipped with a professional wall-climbing facility: intermediate-level athlete on Task $\beta$ (unwrapped, radians). 

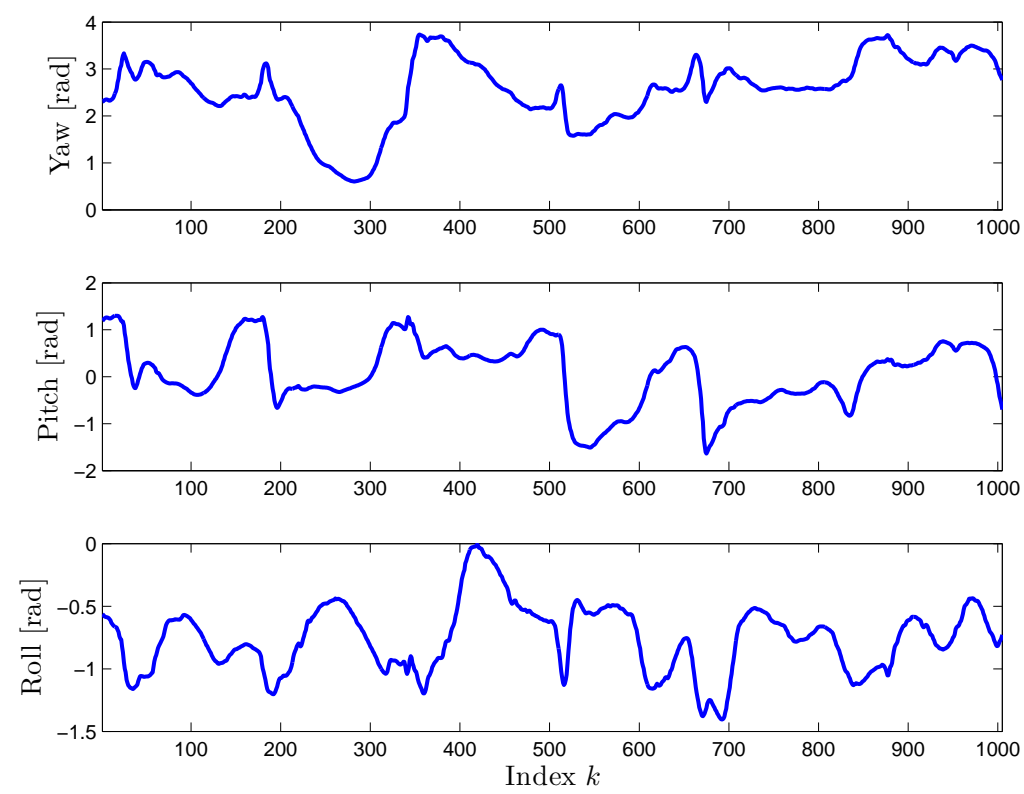

Figure 9. Gyroscopic signals acquired in a gym equipped with a professional wall-climbing facility: expert athlete on Task $\beta$ (unwrapped, radians).

The results of Cartesian kinematic jerk computation are displayed in Table 2. Even in this case, for each task separately, the Cartesian kinematic jerk grows in value as the analyzed motion becomes less fluent. It is interesting to compare the value of the accumulated kinematic jerk $\tilde{J}$ index with the value of the accumulated velocity $\tilde{d}$ also reported in Table 2: there exists a weak correlation between the velocity index and the kinematic jerk index, in general. This experimental observation reflects a known statistical phenomenon, namely, there exists only a weak correlation between a stochastic variable and its derivatives.

Table 2. Distance (12) and Cartesian kinematic jerk index (9) calculated on acquired gyroscopic signals for "Inexpert", "Intermediate-level" and "Expert" athletes on both "Task $\alpha$ " and "Task $\beta$ ".

\begin{tabular}{ccc}
\hline Experiment & Distance (12) & Kin. Jerk Index (9) \\
\hline Inexpert-Task $\alpha$ & 78 & $3,190,177$ \\
Intermediate-level-Task $\alpha$ & 28 & $1,136,228$ \\
Expert-Task $\alpha$ & 41 & 695,616 \\
Inexpert-Task $\beta$ & 39 & 797,533 \\
Intermediate-level-Task $\beta$ & 24 & 674,140 \\
Expert-Task $\beta$ & 22 & 377,639 \\
\hline
\end{tabular}

\section{Conclusions}

The present research work deals with a dedicated acquisition procedure to evaluate hip orientation fluency during a complex body motion task. The illustrated data-acquisition procedure is based on a smartphone endowed with a gyroscopic sensor that is able to reveal the instantaneous orientation of the hips of an athlete during motion. The smartphone runs an application that sends out the measured data over a wireless network and that transmits the acquired data, according to a User Datagram Protocol, to a personal computer.

The personal computer, through a computer program written in MATLAB ${ }^{\circledR}$ language and interpreted by a MATLAB ${ }^{\circledR}$ environment, reads and stores the gyroscopic data-stream and evaluates numerically a kinematic jerk index, taken as a meaningful measure of body movement fluency. The illustrated procedure 
was first tested on specific motor tasks, such as walking, running and jumping, as a validation stage. Then, data acquisitions were performed in a professional gymnasium, equipped with a professional wall-climbing facility. In particular, the hips orientation during climbing of three athletes with different skills were recorded over two different walls.

The obtained results confirm that the procedure is reliable and the numerical results of kinematic jerk index estimation could confirm the self-evaluation of the athletes' skills.

Possible extensions of the present work are real-time processing of the acquired gyroscopic data (to allow athletes to obtain a real-time feedback on their performances), multichannel acquisition by several concurring smartphones (to allow merging gyroscopic data pertaining to different body movements), remote acquisition/processing of gyroscopic data (to allow a central server to acquire and process the data streams sent over a wireless or cable network from different remote geographical sites) in an eHealth setting, and the design of an automated (possibly machine-learning based) classification algorithm to discern between different body-movement fluency levels.

Author Contributions: Conceptualization, S.F.; Software, A.C. and G.R.

Funding: This research received no external funding.

Acknowledgments: The authors wish to thank Ludovic Seifert for sharing his papers on motion fluency estimation, Edoardo Russo and Nicola Sabino, from the School of Information and Automation Engineering, Università Politecnica delle Marche, and Hwee Kuan Lee from the Bio-Informatics Institute (A*STAR, Republic of Singapore), for constructive discussions during the development of the present project.

Conflicts of Interest: The authors declare no conflict of interest.

\section{References}

1. Biess, A.; Liebermann, D.G.; Flash, T. A computational model for redundant human three-dimensional pointing movements: Integration of independent spatial and temporal motor plans simplifies movement dynamics. J. Neurosci. 2007, 27, 13045-13064. [CrossRef] [PubMed]

2. Russell, S.D.; Zirker, C.A.; Blemker, S.S. Computer models offer new insights into the mechanics of rock climbing. Sports Technol. 2012, 5, 120-131. [CrossRef]

3. Seifert, L.; Orth, D.; Boulanger, J.; Dovgalecs, V.; Hérault, R.; Davids, K. Climbing skill and complexity of climbing wall design: Assessment of jerk as a novel indicator of performance fluency. J. Appl. Biomech. 2014, 30, 619-625. [CrossRef] [PubMed]

4. Kerr, A.; Pomeroy, V.P.; Rowe, P.J.; Dall, P.; Rafferty, D. Measuring movement fluency during the sit-to-walk task. Gait Posture 2013, 37, 598-602. [CrossRef] [PubMed]

5. Seifert, L.; Orth, D.; Mantel, B.; Boulanger, J.; Hérault, R.; Dicks, M. Affordance realization in climbing: Learning and transfer. Front. Psychol. 2018, 9, 280.10.3389/fpsyg.2018.00820. [CrossRef] [PubMed]

6. Gouttebarge, V.; Inklaar, H.; Backx, F.; Kerkhoffs, G. Prevalence of osteoarthritis in former elite athletes: A systematic overview of the recent literature. Rheumatol. Int. 2015, 35, 405-418. [CrossRef] [PubMed]

7. Cordier, P.; Dietrich, G.; Pailhous, J. Harmonic analysis of a complex motor behavior. Hum. Mov. Sci. 1996, 15, 789-807. [CrossRef]

8. Sibella, F.; Frosio, I.; Schena, F.; Borghese, N.A. 3D analysis of the body center of mass in rock climbing. Hum. Mov. Sci. 2007, 26, 841-852. [CrossRef] [PubMed]

9. Kettunen, J.A.; Kujala, U.M.; Räty, H.; Videman, T.; Sarna, S.; Impivaara, O.; Koskinen, S. Factors associated with hip joint rotation in former elite athletes. Br. J. Sports Med. 2000, 34, 44-48. [CrossRef] [PubMed]

10. Flash, T.; Hogans, N. The coordination of arm movements: An experimentally confirmed mathematical model. J. Neurosci. 1985, 5, 1688-1703. [CrossRef]

11. Rubio, F.; Valero, F.; Sunyer, J.; Cuadrado, J. Optimal time trajectories for industrial robots with torque, power, jerk and energy consumed constraints. Ind. Robot Int. J. 2012, 39, 92-100. [CrossRef] 
12. Tack, G.-R.; Choi, J.S.; Yi, J.H.; Kim, C.H. Relationship between jerk cost function and energy consumption during walking. In Proceedings of the 2006 World Congress on Medical Physics and Biomedical Engineering, Seoul, Korea, 27 August-1 September 2006; Volume 14, pp. 2917-2918.

13. He, H.; Li, R.; Chen, K. Characteristics of jerk response spectra for elastic and inelastic systems. Shock Vib. 2015, 2015, 782748. [CrossRef]

14. Birt, J.; Stromberga, Z.; Cowling, M.; Moro, C. Mobile mixed reality for experiential learning and simulation in medical and health sciences education. Information 2018, 9, 31.10.3390/info9020031. [CrossRef]

15. Van, D.D.; Ai, Q.; Liu, Q. Vertical handover algorithm for WBANs in ubiquitous healthcare with quality of service guarantees. Information 2017, 8, 34.10.3390/info8010034. [CrossRef]

16. Mellone, S.; Tacconi, C.; Chiari, L. Validity of a smartphone-based instrumented timed up and go. Gait Posture 2012, 36, 163-165. [CrossRef]

17. Nishiguchi, S.; Yamada, M.; Nagai, K.; Mori, S.; Kajiwara, Y.; Sonoda, T.; Yoshimura, K.; Yoshitomi, H.; Ito, H.; Okamoto, K.; et al. Reliability and validity of gait analysis by android-based smartphone. Telemed. e-Health 2012, 18, 292-296. [CrossRef]

18. Kim, A.; Kim, J.; Rietdyk, S.; Ziaie, B. Field Assessment of Gait: Accurate Measures of Step Length and Step Length Variability Provided With a Simple, Inexpensive Device. June 2014. Available online: http: / /www.purdue.edu/ newsroom/releases/2014/Q2/smartphone-adapted-to-measure-persons-gait,-reduce-falls.html (accessed on 11 December 2018).

19. Fujinami, K. On-body smartphone localization with an accelerometer. Information 2016, 7, 21. [CrossRef]

20. Camurri, A.; Mazzarino, B.; Ricchetti, M.; Timmers, R.; Volpe, G. Multimodal analysis of expressive gesture in music and dance performances. In Gesture-Based Communication in Human-Computer Interaction (GW 2003); Lecture Notes in Computer Science; Springer: Berlin/Heidelberg, Germany, 2003; Volume 2915.

21. Uemura, M.; Tomikawa, M.; Kumashiro, R.; Miao, T.; Souzaki, R.; Ieiri, S.; Ohuchida, K.; Lefor, A.T.; Hashizume, M. Analysis of hand motion differentiates expert and novice surgeons. J. Surg. Res. 2014, 188, 8-13. [CrossRef]

22. Klaas, V.C.; Troster, G.; Walt, H.; Jenewein, J. Remotely monitoring cancer-related fatigue using the smart-phone: Results of an observational study. Information 2018, 9, 271. [CrossRef]

23. Seifert, L.; Cordier, R.; Orth, D.; Courtine, Y.; Croft, J.L. Role of route previewing strategies on climbing fluency and exploratory movements. PLoS ONE 2017, 12, e0176306.10.1371/journal.pone.0176306. [CrossRef]

24. Hyper IMU Mobile App. Available online: https://play.google.com/store/apps/details?id=com.ianovir.hyper_ imu (accessed on 11 December 2018).

25. Postel, J. RFC 768: User Datagram Protocol. Internet Engineering Task Force. August 1980. Available online: https: / tools.ietf.org/html/rfc768 (accessed on 11 December 2018).

26. Greenwood, D.T. Principles of Dynamics, 2nd ed.; Prentice Hall: Upper Saddle River, NJ, USA, 1988.

27. Chirikjian, G.S. Stochastic Models, Information Theory, and Lie Groups, Volume 2: Analytic Methods and Modern Applications, 1st ed.; Birkhäuser: Basel, Switzerland, 2011.

28. Tsao, C.-C.; Mirbagheri, M.M. Upper limb impairments associated with spasticity in neurological disorders. J. NeuroEng. Rehabil. 2007, 4, 1-15. [CrossRef] [PubMed]

29. Edelman, A.; Arias, T.A.; Smith, S.T. The geometry of algorithms with orthogonality constraints. SIAM J. Matrix Anal. Appl. 1998, 20, 303-353. [CrossRef]

30. Fiori, S.; Tanaka, T. An algorithm to compute averages on matrix Lie groups. IEEE Trans. Signal Process. 2009, 57, 4734-4743. [CrossRef]

31. Saha, J.; Chowdhury, C.; Chowdhury, I.R.; Biswas, S.; Aslam, N. An ensemble of condition based classifiers for device independent detailed human activity recognition using smartphones. Information 2018, 9, 94. [CrossRef]

32. Timmins, K.A.; Leech, R.D.; Batt, M.E.; Edwards, K.L. Running and knee osteoarthritis: A systematic review and meta-analysis. Am. J. Sports Med. 2017, 45, 1447-1457. [CrossRef]

(C) 2018 by the authors. Licensee MDPI, Basel, Switzerland. This article is an open access article distributed under the terms and conditions of the Creative Commons Attribution (CC BY) license (http:/ / creativecommons.org/licenses/by/4.0/). 